\title{
manomanum \\ Oncolytic vaccinia virus shows promise in liver cancer
}

The use of oncolytic immunotherapy for treating cancer has generated much interest in recent years. Oncolytic viruses are designed to specifically replicate in and destroy tumour cells while inducing tumour-specific immunity. The vaccinia virus JX-594 consists of a disrupted thymidine kinase gene for cancer selectivity, the human granulocytemacrophage colony-stimulating factor (hGM-CSF) transgene for immune stimulation fused to a reporter $\beta$-galactosidase transgene. JX-594 has shown positive results in terms of efficacy and tolerability in phase I trials. Now, two phase II trials using JX-594 to treat patients with advanced hepatocellular carcinoma (HCC) have been published.

The first trial was a randomized phase II study that compared low-dose $\left(10^{8} \mathrm{PFU}\right)$ versus high dose $\left(10^{9} \mathrm{PFU}\right)$ JX-594 treatment in 30 patients with HCC. Therapy was generally welltolerated at both doses with no treatmentrelated deaths reported. Intrahepatic tumour response was assessed using modified RECIST criteria (mRECIST) developed for patients with HCC.

At the primary radiographic end point of 8 weeks, the intrahepatic mRECIST disease control rate was comparable for the high-dose and low-dose groups. Modified Choi response criteria was used to assess effects on perfusion and tumour necrosis. Similar outcomes between the two groups were observed; in addition, for both doses, comparable outcomes were seen for tumours injected with JX-594 and distant tumours at noninjected sites.

Viral replication was assessed by measuring expression of the hGM-CSF and $\beta$-galactosidase transgenes. Expression of hGM-CSF was detectable in $69 \%$ of patients in the high-dose group and $46 \%$ of patients in the low-dose group on day 5 of the trial, indicative of viral replication. This was followed by the induction of antitumoural immunity. Despite similar tumour responses with both doses, median overall survival was longer in the high-dose compared with the low-dose group (14.1 months versus 6.7 months).

The second trial evaluated if JX-594 induced tumour vascular disruption in patients with HCC. The researchers first showed that JX-594 could infect and lyse VEGF and FGF-2 activated human endothelial cells in vitro, and that it could disrupt tumour vasculature in mouse tumour models by selectively reducing blood flow to solid tumours.

Tumour perfusion was subsequently evaluated in 15 patients with HCC in a phase II trial of JX-594. MRI scans showed that tumour perfusion decreased significantly over time in injected and noninjected tumours. In tumours injected with JX-594, a decrease in perfusion of $15 \%$ or more was seen in $33 \%$ of patients 5 days after injection. Similar results were observed in noninjected tumours. The vascular disruption was maintained for at least 8 weeks, with no signs of damage to normal vasculature observed.

These trials confirm that, in HCC, JX-594 has antitumour efficacy, effects on the tumour vasculature, and a dosedependent effect on overall survival.

Vanessa Marchesi

Original articles $\mathrm{Heo}$, J. et al. Randomized dosefinding clinical trial of oncolytic immunotherapeutic vaccinia JX-594 in liver cancer. Nat. Med. doi:10.1038/ nm.3089 | Breitbach, C. J. et al. Oncolytic vaccinia virus disrupts tumor-associated vasculature in humans. Cancer Res. doi:10.1158/0008-5472.CAN-12-2687 